\title{
ASO Author Reflections: Magnetic Seed Localization and Tracers Add Value to Multidisciplinary Breast Programs
}

\author{
Megan E. Miller, MD, FACS, and Jill Dietz, MD, FACS \\ Department of Surgery, University Hospitals Cleveland Medical Center, Case Western Reserve University School of \\ Medicine, Cleveland, $\mathrm{OH}$
}

\section{PAST}

While multiple methods to replace traditional wire localization for breast cancer surgery have been studied, the challenge for surgeons is to select and implement the technology best suited to each hospital system and practice. With the largest published, nonradioactive wireless seed localization experience, we demonstrated that magnetic seeds can be used to localize breast lesions and lymph nodes safely and accurately, for subsequent removal. ${ }^{1}$ Furthermore, this technique dissociates the localization procedure from surgery, resulting in on-time case starts and improved patient and user experience. Operative procedures are now performed at less expensive ambulatory surgery centers without radiology services. These benefits have resulted in almost complete replacement of wires for localization in our hospital system.

\section{PRESENT}

During the COVID-19 pandemic, it is imperative to limit patient and staff exposures, particularly in the hospital setting. Scheduled magnetic seed localization on the day of surgeon consultation or preoperative testing reduces

This is an invited commentary submitted as an ASO Author Reflection. The accompanying study was presented in poster format at the American Society of Breast Surgeons 20th Annual Meeting, April 30 to May 5, 2019, Dallas, TX.

(C) Society of Surgical Oncology 2020

First Received: 3 November 2020

Accepted: 3 November 2020;

Published Online: 23 November 2020

M. E. Miller, MD, FACS

e-mail: Megan.Miller6@UHhospitals.org contact risk and has become our routine. On the day of surgery, patients need not travel for localization to another area of the hospital, further limiting potential exposure. As our reimbursement system transitions from fee-for-service to value-based payment models and the need for consolidated care increases, magnetic seeds can be placed at time of biopsy for category $\mathrm{V}$ lesions, eliminating the additional localization procedure. This is particularly valuable in axillary lymph nodes where studies have shown that localization of positive nodes decreases false-negative rates by ensuring removal during sentinel lymph node (SLN) biopsy. ${ }^{2,3}$ Additionally, our study showed that localization and removal of lymph nodes that were suspicious by imaging but with negative pathology on core needle biopsy, actually yielded malignant cells in $17.4 \%$ of these localized nodes on surgical pathology. ${ }^{1}$ This unexpected false-negative rate in suspicious-appearing nodes with benign pathology has altered our practice such that we localize and remove all previously biopsied lymph nodes during axillary surgery. With the increasing use of neoadjuvant therapy, magnetic seed localization at time of biopsy provides the best value and accuracy for future node retrieval.

\section{FUTURE}

The ultimate in improved value is to eliminate unnecessary procedures altogether. In addition to magnetic seed localization, we initiated the use of Magtrace, an iron oxide nanoparticle dual tracer, for SLN identification. This tracer uses the same detection equipment in the operating theater as magnetic seeds, eliminating the need for a second probe system and its associated cost. The unique property of this tracer is that after injection, it remains in the lymph nodes for weeks and, unlike other tracers, allows for delayed SLN biopsy. ${ }^{4}$ Patients with ductal carcinoma in situ (DCIS) 
undergoing mastectomy or large oncoplastic procedures can have Magtrace injected at the time of primary surgery but avoid SLN biopsy. Only the patients who upgrade to invasive disease require a return to the operating room for node excision, saving thousands of women per year the cost and complication risks associated with SLN biopsy. ${ }^{5}$ As technology evolves, we will likely be able to place both seeds and tracer for patients with invasive disease at the time of biopsy or the day of surgery, limiting additional procedures. In the operating room, using the same equipment on different settings, we will localize the breast lesion and previously biopsied lymph nodes using magnetic seeds, and identify the SLNs simultaneously using magnetic tracer. Moving forward, the use of magnetic seeds and tracers will allow us to provide a better patient experience more efficiently, adding value to our patient's breast cancer journey.

DISCLOSURES The authors have no conflicts of interest to disclose.

\section{REFERENCES}

1. Miller ME, Nirav P, Freyvogel M, et al. Hospital system adoption of magnetic seeds for wireless breast and lymph node localization. Ann Surg Oncol. 2020. https://doi.org/10.1245/s10434-020-09311$\mathrm{x}$.

2. Boughey JC, Ballman KV, Le-Petross HT, et al. Identification and resection of clipped node decreases the false-negative rate of sentinel lymph node surgery in patients presenting with nodepositive breast cancer (T0-T4, N1-N2) who receive neoadjuvant chemotherapy. Ann Surg. 2016;263:802-7.

3. Caudle A, Yang W, Krishnamurthy S, et al. Improved axillary evaluation following neoadjuvant therapy for patients with nodepositive breast cancer using selective evaluation of clipped nodes: implementation of targeted axillary dissection. J Clin Oncol. 2016;34:1072-8.

4. Karakatsanis A, Hersi A-F, Pistolis L, et al. Effect of preoperative injection of superparamagnetic iron oxide particles on rates of sentinel lymph node dissection in women undergoing surgery for ductal carcinoma in situ (SentiNot study). $\mathrm{Br} \mathrm{J}$ Surg. 2019;106(6):720-8.

5. Ashikaga T, et al. Morbidity results from the NSABP B-32 trial comparing sentinel lymph node dissection versus axillary dissection. J Surg Oncol. 2010;102(2):111-8.

Publisher's Note Springer Nature remains neutral with regard to jurisdictional claims in published maps and institutional affiliations. 